\title{
Mathematical Morphology in any color space
}

\author{
O. Lezoray, A. Elmoataz \\ LUSAC EA 2607, IUT SRC, \\ 120 rue de l'exode, F-50000 Saint-Lô, FRANCE
}

\author{
C. Meurie \\ INRETS-LEOST, 20 rue Elisee Reclus \\ BP 317, 59666 Villeneuve d'ascq, FRANCE
}

\begin{abstract}
In this paper, a new graph-based ordering of color vectors is presented for mathematical morphology purposes. An attractive propoerty of the proposed ordering is its color space independence. A complete graph is defined over a filter window and its structure is analyzed to construct an ordering of color vectors by finding a Hamiltonian path in a two-step algorithm.
\end{abstract}

\section{Introduction}

A multivariate image can be represented by the mapping $\mathbb{Z}^{l} \rightarrow \mathbb{R}^{p}$ where $l$ is the image dimension and $p$ the number of channels. Let $W=\left\{\mathbf{x}_{k} \in \mathbb{Z}^{l} ; k=1,2, \ldots, N\right\}$ design a filter window of finite length $N$ where $\mathbf{x}_{1}$ determines the position of the filter window. From $W$, one therefore gets a set $\left\{\mathbf{x}_{1}, \mathbf{x}_{2}, \cdots, \mathbf{x}_{N}\right\}$ of $N p$-dimensional vectors: $\mathbf{x}_{i}=\left\{\mathbf{x}_{i}^{1}, \mathbf{x}_{i}^{2}, \cdots, \mathbf{x}_{i}^{p}\right\}, \mathbf{x}_{i} \in \mathbb{R}^{p}$. A classical way to define an ordering relation between vectors is to use a transform [6] $h$ from $\mathbb{R}^{p}$ into $\mathbb{R}^{q}$ followed by the natural ordering on each dimension of $\mathbb{R}^{q}$. With $h: \mathbb{R}^{p} \rightarrow \mathbb{R}^{q}$, and $\mathbf{x} \rightarrow h(\mathbf{x})$ then $\forall\left(\mathbf{x}_{i}, \mathbf{x}_{j}\right) \in \mathbb{R}^{p} \times \mathbb{R}^{p}, \mathbf{x}_{i} \leq \mathbf{x}_{j} \Leftrightarrow h\left(\mathbf{x}_{i}\right) \leq h\left(\mathbf{x}_{j}\right)$. When $h$ is bijective, this corresponds to define a space filling curve that goes through each point of the $\mathbb{R}^{p}$ space just once and thus induces a total ordering. Therefore, there is an equivalence: (total ordering on $\left.\mathbb{R}^{p}\right) \Leftrightarrow$ (bijective application $\left.h: \mathbb{R}^{p} \rightarrow \mathbb{R}\right) \Leftrightarrow\left(\right.$ space filling curve in $\mathbb{R}^{p}$ ) $[14,4]$. When the space is represented by a connected graph, we point out (in the next section) another equivalence: (space filling curve in $\left.\mathbb{R}^{p}\right) \Leftrightarrow\left(\right.$ Hamiltonian path on $\left.\mathbb{R}^{p}\right)$.

Mathematical morphology is a nonlinear approach to image processing which relies on a fundamental structure, the complete lattice $\mathcal{L}$ [12]. An ordering relation $\leq$ is defined over $\mathcal{L}$ and for every finite subset $\mathcal{K}$ of $\mathcal{L}$, there exists a supremum $\vee \mathcal{K}$ and an infimum $\wedge \mathcal{K}$. Therefore, Mathematical morphology needs total orderings [15]. The classical way to perform morphological operations is then to use a conditional ordering where the vectors are ordered according to a hierarchical order of the components [2]. This or- dering is a total ordering of vectors but it introduces a strong dissymmetry between the components. The choice of the priority component being difficult [2], this can be alleviated by considering perceptual color spaces based on Luminance/Hue/Saturation $[2,9]$ where the ordering is more natural from a human perception point of view. However, this does not enable to directly perform morphological operations in any color space even if if conditional ordering can be combined with a reduced ordering [10] based on distances [3]. In this paper, we propose a graph-based ordering of color vectors which does not make any assumption on the color space under consideration [8].

\section{Graph-Based ordering of color vectors}

\subsection{Preliminaries on graphs}

We provide some basic definitions on graph theory [5]. A graph $\mathcal{G}$ is a couple $\mathcal{G}=(V, E)$ where $V$ is a finite set of vertices and $E$ is a set of edges included in a subset of $V \times V$. Two vertices $u$ and $v$ in a graph are adjacent if the edge $(u, v)$ exists in $E$. The degree $\delta(v)$ of a vertex $v$ is the number of edges incident to the vertex. $\delta: V \rightarrow \mathbb{N}$ is defined as $\delta(v)=|u \sim v|$ where $|\cdot|$ denotes the cardinal of a set. $u \sim v$ denotes the set of vertices $u$ connected to the vertex $v$ via the edges $(u, v) \in E$. A path $p$ is a set of vertices $p=\left(v_{1}, v_{2}, \cdots, v_{k}\right)$ such as there is an edge for each two successive vertices of the path: $\forall i \in[1, k[$, the edge $\left(v_{i}, v_{i+1}\right) \in E$. The length of a path corresponds to its number of edges. A path is Hamiltonian if it uses all the vertices exactly once (this problem is NP-complete). A complete graph is a graph where an edge connects every pair of vertices. A complete graph with $n$ vertices has $n(n-$ $1) / 2$ edges and the degree of each vertex is $(n-1)$. A graph is connected when for every pair of vertices $u$ and $v$ there is a path in which $v_{1}=u$ and $v_{k}=v$. In the rest of this paper, we consider only simple graphs for which maximum one edge can link two vertices. These simple graphs are always assumed to be connected and undirected [5]. A graph, as defined above, is said to be weighted if it is associated with a weight function $w: E \rightarrow \mathbb{R}^{+}$satisfying 
$w(u, v)>0$ if $(u, v) \in E, w(u, v)=0$ if $(u, v) \notin E$. We can now define the space of functions on graphs. Let $\mathcal{H}(V)$ denote the Hilbert space of real-valued functions on vertices, in which each $f: V \rightarrow \mathbb{R}^{+}$assigns a real value $f(v)$ to each vertex $v$. A function $f$ in $\mathcal{H}(V)$ can be thought as a column vector in $\mathbb{R}^{|V|}$. Similarly, one can define $\mathcal{H}(E)$ the space of real-valued functions on edges, in which each one $g: E \rightarrow \mathbb{R}^{+}$assigns a real value to each edge $e$.

A tree is a connected acyclic simple graph. A spanning tree of a connected, undirected graph $\mathcal{G}$ is a tree composed of all the vertices and some of the edges of $\mathcal{G}$. A minimum spanning tree (MST) is then a spanning tree with weight less than the weight of every other spanning tree. Therefore, a minimum spanning tree $\mathcal{T}(\mathcal{G})$ of a graph $\mathcal{G}$ is a weighted connected graph $\mathcal{T}(\mathcal{G})=\left(V^{\prime}, E^{\prime}\right)$ where the sum of the weights $\sum_{(u, v) \in E^{\prime}} w(u, v)$ is minimum. For a graph $\mathcal{G}$ of $n$ vertices, its $\operatorname{MST} \mathcal{T}(\mathcal{G})$ has exactly $(n-1)$ edges.

\subsection{Graph-based ordering: bounds extraction}

In this paper, we take a Hamiltonian path point of view of the ordering of vectors. However, we propose to dynamically construct such a Hamiltonian path on a filter window $W$ rather on the complete space $\mathbb{R}^{p}$. To a given filter window $W$, we can associate a complete graph $\mathcal{G}_{0}$ the vertices of which correspond to the vectors of $W$. This corresponds to a function $f \in \mathcal{H}(V), f: V \rightarrow W$ which associates a color vector $\mathbf{x} \in W$ to each vertex. Similarly, we can associate a weight to each edge of the graph, $w \in \mathcal{H}(E), w: E \rightarrow \mathbb{R}^{+}$. Classically, we consider $w(u, v)=\|f(u)-f(v)\|_{2}$. Since it is difficult to find an optimal Hamiltonian path on $\mathcal{G}_{0}$ among all the $(|W|-1)$ ! different possibilities, we propose to approximate this path. Instead of trying to directly define the Hamiltonian path, we begin by extracting its bounds $(\wedge$ and $\vee)$. Let $\mathcal{T}_{0}=\mathcal{T}\left(\mathcal{G}_{0}\right)$ denote the MST of $\mathcal{G}_{0}$. An MST being a generalization to higher dimensions of a one-dimension sorted list, we can use its structure to find candidate bounds of the Hamiltonian path. A vertex $v$ of a Hamiltonian path is one of its bounds iff $\delta(v)=1$ : it is a leaf. We use this principle to extract them [7]. Let $\mathcal{N}_{0}=\left\{u \mid \delta(u)=1, u \in \mathcal{T}_{0}\right\}$ denote the leaves of $\mathcal{T}_{0}$. The vertices in $\mathcal{N}_{0}$ are the only candidates for bounds of the Hamiltonian path. Since most of the time $\left|\mathcal{N}_{0}\right|>2, \mathcal{N}_{0}$ has to be reduced to only two elements. To that aim, we iterate the same process on the complete graph constructed over the vertices of $\mathcal{N}_{0}$ until $\left|\mathcal{N}_{i}\right|=2$ with $i$ the iteration number. To sum it up, to extract the bounds of a Hamiltonian path, the principle is as following:

$i \leftarrow 0 ; \mathcal{G}_{i}$ : the complete graph over filter window $W$

\section{Repeat}

$\mathcal{T}_{i}=\mathcal{T}\left(\mathcal{G}_{i}\right) ; \mathcal{N}_{i}=\left\{u \mid \delta(u)=1, u \in \mathcal{T}_{i}\right\}$

$\mathcal{G}_{i+1}$ : the complete graph over over $\mathcal{N}_{i} ; i \leftarrow i+1$

Until $\left|\mathcal{N}_{i-1}\right|=2$
$\mathcal{T}_{i}=\mathcal{G}_{i}, \mathcal{N}_{i}=\left\{u \in \mathcal{T}_{i}\right\}$

At the end of the process, $\mathcal{N}_{i}=\{u, v\}$ contains two vertices considered as bounds of the Hamiltonian path. However, one still has to define which one of these two vertices is the $\vee$ (respectively the $\wedge$ ). The $\wedge$ is identified as the closest vertice to a reference color $\mathbf{x}_{r e f}$ [13] which is usually black: $\vee=\arg \max _{v \in \mathcal{N}_{i}}\left\|f(v), \mathbf{x}_{\text {ref }}\right\|_{2}$ and $\wedge=$ $\arg \min _{v \in \mathcal{N}_{i}}\left\|f(v), \mathbf{x}_{\text {ref }}\right\|_{2}$. One then can prove that the complexity of this step is $O\left(N^{3} \log N\right)$. Figure 1 presents the

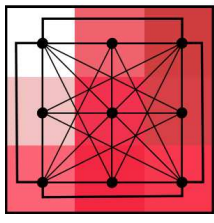

(a) $\mathcal{G}_{0}$

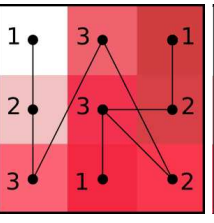

(b) $\mathcal{T}_{0}$

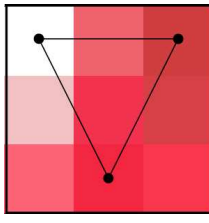

(c) $\mathcal{G}_{1}$

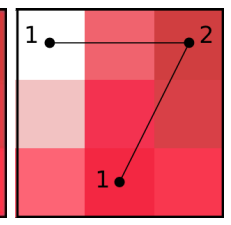

(d) $\mathcal{T}_{1}$
Figure 1. The graphs $\mathcal{G}_{i}$ and $\mathcal{T}_{i}$ in the different steps of the algorithm for Hamiltonian path bounds extraction. For each $\mathcal{T}_{i}$, the vertices degrees are superimposed. $\mathcal{G}_{2}$ and $\mathcal{T}_{2}$ are not shown here.

different steps of the algorithm. The complete graph $\mathcal{G}_{0}$ is constructed over the filter window $W$ (Figure 1(a)) and its MST $\mathcal{T}_{0}$ is computed (Figure 1(b)). A new complete graph $\mathcal{G}_{1}$ (Figure $1(\mathrm{c})$ ) is constructed over the leaves of $\mathcal{T}_{0}$ and its MST $\mathcal{T}_{1}$ is computed (Figure $1(\mathrm{~d})$ ). Finally, A complete graph $\mathcal{G}_{2}$ of two vertices is obtained. With $\mathbf{x}_{\text {ref }}$ as black, the $\vee$ is the top left pixel of $W$ and $\wedge$ the bottom middle one.

\subsection{Graph-based ordering: Ordering construction}

Once the two bounds of the Hamiltonian path have been determined, the complete Hamiltonian path can be constructed. On the filter window $W$ under consideration, a Hamiltonian path $p=\left(v_{1}, v_{2}, \cdots, v_{k}\right)$ has a length of $|W|$ and its bounds are $v_{1}=\wedge$ and $v_{k}=\vee$. The construction of the Hamiltonian path we propose is based on the nearest neighbor principle on the initial complete graph $\mathcal{G}_{0}$. To determine the nearest neighbor $u$ of a vertex $v$, we consider the weight of the edge $w(u, v)$ but also the saliency of the neighbor $u$ in the graph. The saliency of a vertex quantifies its global importance in the set of MST which where generated during the inf and sup extraction. It is defined as follows: $\Delta(v)=\sum_{i=0}^{i_{\max }}(i+1) \cdot \delta\left(\mathcal{T}_{i}, v\right)$ where $\delta\left(\mathcal{T}_{i}, v\right)=\delta(v), v \in \mathcal{T}_{i}$ where $i$ corresponds to an iteration number in the Hamiltonian path bounds extraction and $i_{\max }$ to the total number of iterations. The bounds $v_{1}$ and $v_{N}$ have the highest saliences. Otherwise, the saliency of 
a vertex is all the more important when it survives in the successive $\mathcal{T}_{i}$. For instance, the saliency of the top left pixel $v_{j}$ of the filter window in Figure 1(a) is $\Delta\left(v_{j}\right)=$ $1 \cdot \delta\left(\mathcal{T}_{0}, v_{j}\right)+2 \cdot \delta\left(\mathcal{T}_{1}, v_{j}\right)+3 \cdot \delta\left(\mathcal{T}_{2}, v_{j}\right)=1 \cdot 1+2 \cdot 1+3 \cdot 1=6$. The construction of the Hamiltonian path is $O\left(N^{2}\right)$ and can then be summarized as follows:

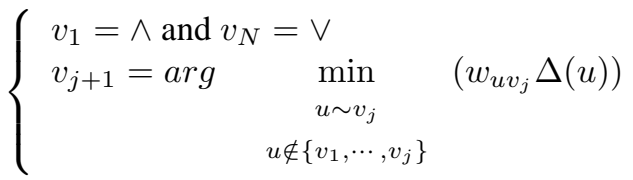
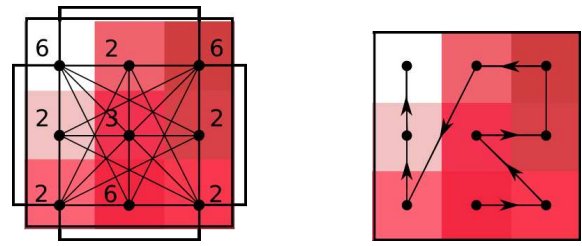

Figure 2. Left: graph $\mathcal{G}_{0}$ with vertices saliences $\Delta(v)$ superimposed. Right: constructed Hamiltonian path.

Figure 2 illustrates the construction of the ordering of vectors of $W$ as a Hamiltonian path: on the complete graph $\mathcal{G}_{0}$ (Figure 2(a), with vertices saliences superimposed), one obtains the path depicted by figure 2(b).

\section{Results and discussion}

In this section, we illustrate the behavior of the proposed graph-based ordering of color vectors for different mathematical morphology operations. First, erosion $\varepsilon$ and dilatation $\delta$ are considered. These operation retain the infimum (resp. the sup.) of a color set as the output of the filtering. Figure 3 shows the result of an erosion on an image in different color spaces among $R G B, I H S L$ [2], $L^{*} a^{*} b^{*}$ and $C I E C A M 02$ [11]. When needed, the illuminant is considered as $D 65$. On can see that the results visually look quite similar whatever the color space except for CIECAM02. For all color spaces, the reference color was considered as black and this has an influence on the result, another reference color might be more suitable for CIECAM02. It is worth noting that the dimension of a color vector in $C I E C A M 02$ color space is six $(p=6)$ and it will be very difficult to find a proper ordering of the color components for the conditional ordering. In constrast, our graph-based ordering of color vectors can process any color image representation even with high-dimensional vectors as long as a distance measure can be associated to these vectors. We do not consider the properties of the used color spaces and consider color vectors as real-valued vectors. With an ordering of color vectors, we can perform other morphological operations. An usual operator is the morphological gradient $\nabla(f)=\delta(f)-\varepsilon(f)$. Figure 4 presents the obtained morphological gradients in different color spaces for a natural image. The obtained gradients are of course different, but the interest in our graph-based ordering lies in the fact that its computation remains the same whatever the color space. To further illustrate this, Figure 4(f) presents the result of a morphological color contrast mapping [1] on CIECAM02 color vectors. With a computation of the morphological gradient which is color space independent, one can then apply the waterfall algorithm in different color spaces. Figure 5 presents the $4^{\text {th }}$ partition level of the nonparametric hierarchy built by the waterfall algorithm. Saliency maps which give the highest level of appearance of a boundary are also provided. Of course, results differ in the considered color spaces. However, with our graph-based ordering, the ordering has not to be adapted to the color space under study. To our knowledge, this is the first time, that mathematical morphology operations are performed in color spaces of highdimensions such as CIECAM02. Moreover, our graph ordering scheme can be directly applied to the processing of multi-spectral images. However, our graph-based ordering computational complexity is $O\left(N^{3} \log N\right)$ which this is higher than the conditional ordering and our approach is not suited for large filter windows.

\section{Acknowledgements}

This research work was partially supported by the ANR foundation under grant ANR-06-MDCA-00801/FOGRIMMI

\section{References}

[1] J. Angulo. Morphological color processing based on distances. Application to color denoising and enhancement by centre and contrast operators. In Proc. of VIIP, pages 314319, 2005.

[2] J. Angulo. Unified morphological color processing framework in a lum/sat/hue representation. In Proc. of ISMM, pages 387-396, 2005.

[3] J. Angulo. Morphological colour operators in totally ordered lattices based on distances: Application to image filtering, enhancement and analysis. Computer Vision and Image Understanding, In press, 2007.

[4] J. Chanussot and P. Lambert. Total ordering based on space filling curves for multivalued morphology. In Proc. of ISMM, pages 51-58, 1998.

[5] R. Diestel. Graph Theory, volume 173. Springer-Verlag, 2005.

[6] J. K. Goutsias, H. J. A. M. Heijmans, and K. Sivakumar. Morphological operators for image sequences. Computer Vision and Image Understanding, 62(3):326-346, 1995. 

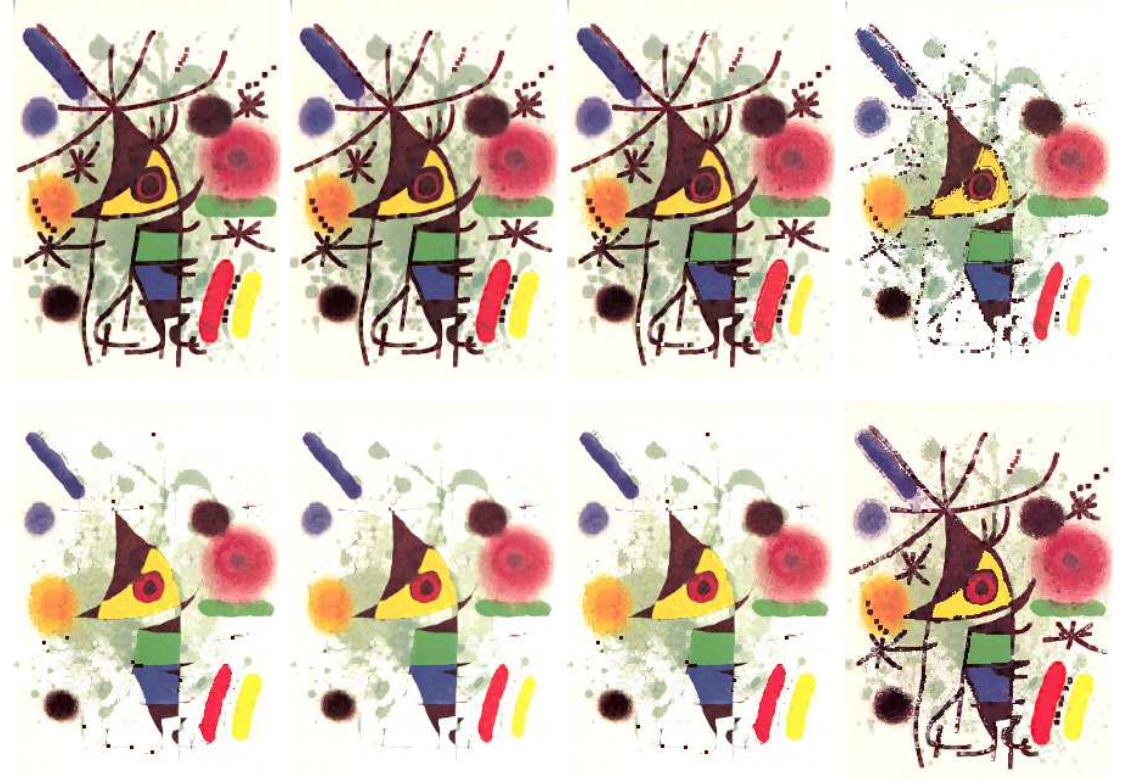

Figure 3. $\varepsilon$ (first row) and $\delta$ (second row) operations in the $R G B, I H S L, L^{*} a^{*} b^{*}$ and $C I E C A M 02$ color spaces (from left to right).

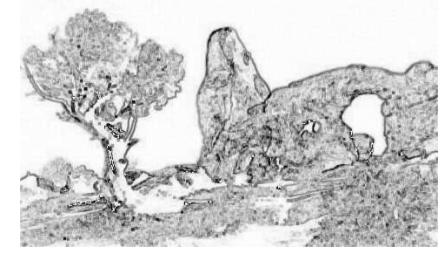

(a) $\nabla_{R G B}$

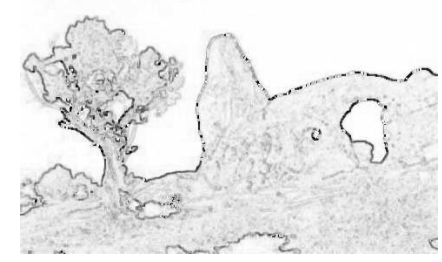

(d) $\nabla_{C I E C A M 02}$

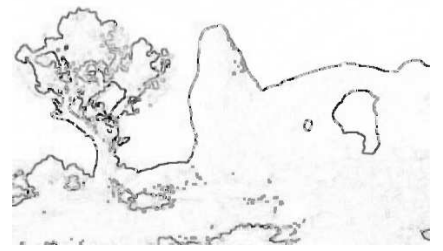

(b) $\nabla_{I H S L}$

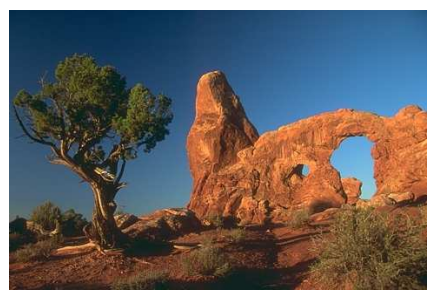

(e) Original image.

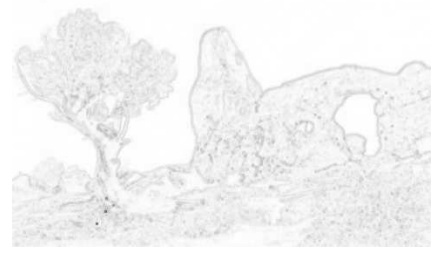

(c) $\nabla_{L^{*} a^{*} b^{*}}$

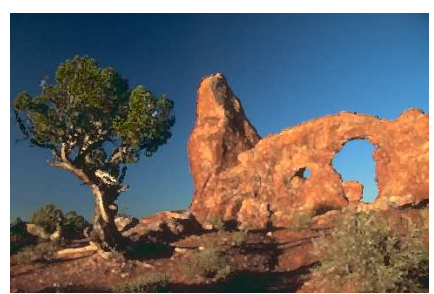

(f) CIECAM02 Morphological contrast mapping.

Figure 4. Morphological gradients (Figures $\mathbf{4 ( a )}$ to $\mathbf{4 ( d )}$ in $R G B, I H S L, L^{*} a^{*} b^{*}$ and $C I E C A M 02$ color spaces) and contrast mapping (Figure 4(f)) in $C I E C A M 02$ color space on a color image (Figure 4(e)). 

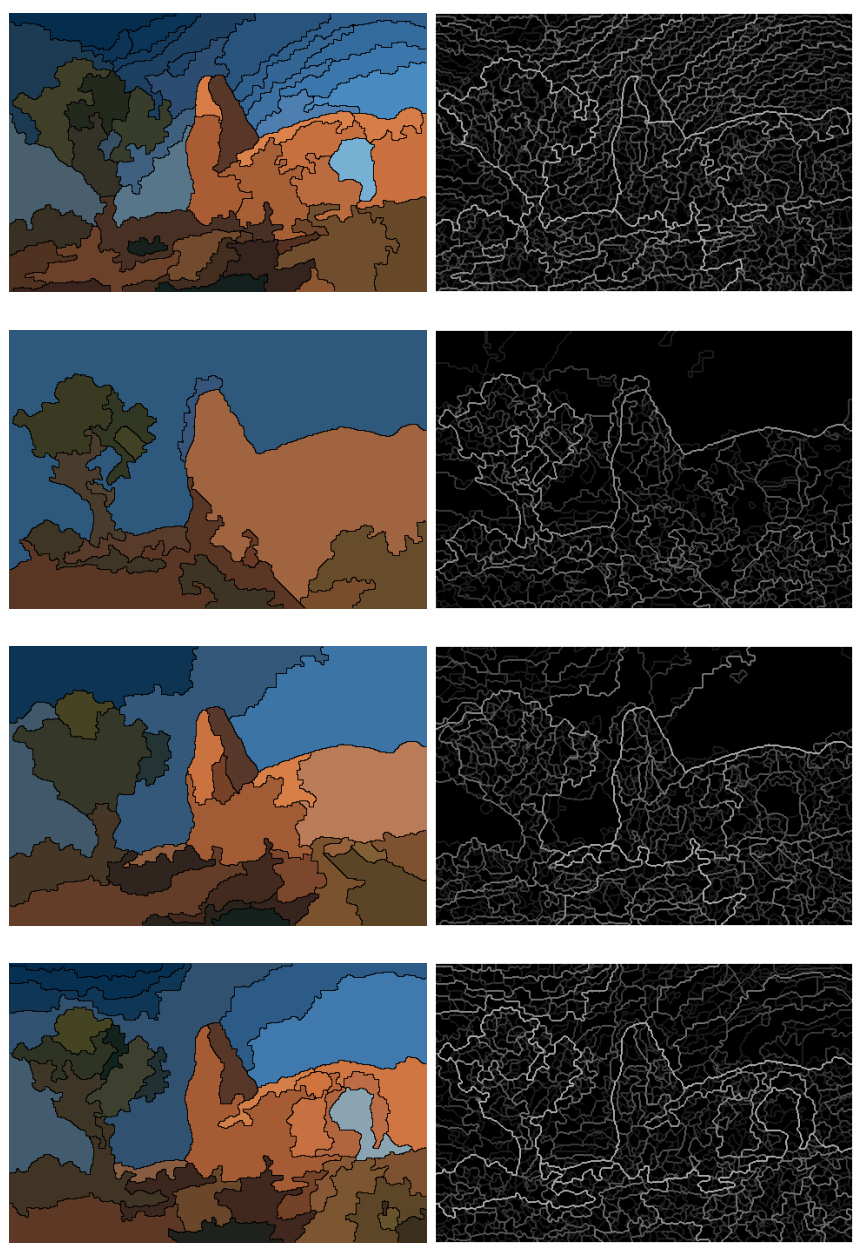

Figure 5. $4^{\text {th }}$ level of a waterfall hierarchy of partitions in several color spaces (first to last row: $\left.R G B, I H S L, L^{*} a^{*} b^{*}, C I E C A M 02\right)$. Each row presents the mosaic image and the saliency map of the whole set of partitions.

[7] O. Lezoray, C. Meurie, and A. Elmoataz. A graph approach to color mathematical morphology. In Proc. of ISSPIT, pages 856-861, 2005.

[8] O. Lezoray, C. Meurie, and A. Elmoataz. Graph-based ordering scheme for color image filtering. International Journal of Image and Graphics, To appear, 2007.

[9] G. Louverdis, M. Vardavoulia, I. Andreadis, and P. Tsalides. A new approach to morphological color image processing. Pattern recognition, 35(8):1733-1741, 2002.

[10] R. Lukac and K. Plataniotis. A taxonomy of color image filtering and enhancement solutions. In P. Hawkes, editor, $A d$ vances in Imaging and Electron Physics, volume 140, pages 187-264. Elsevier, 2006.

[11] N. Moroney, M. Fairchild, R. Hunt, C. Li, M. Luo, and T. Newman. The CIECAM02 color appearance model. In
Proceedings of the IS\&T/SID 10th Color Imaging Conference, 2002.

[12] C. Ronse. Why mathematical mophology needs complete lattices. Signal Processing, 21(2):129-154, 1990.

[13] A. Soria-Frisch and M. Koppen. The fuzzy integral as similarity measure for a new color morphology. In Proc. of CGIV, pages 523-526, 2002.

[14] E. Stringa, A. Teschioni, and C. S. Regazzoni. A classical morphological approach to color image filtering based on space filling curves. In Proceedings of the IEEE-EURASIP Workshop NSIP, pages 351-354, 1999.

[15] H. Talbot, C. Evans, and R. Jones. Complete ordering and multivariate mathematical. In Proceedings of ISMM, pages 27-34, 1998. 